\title{
SOME INEQUALITIES FOR FUNCTIONS HAVING AN $s$-CONVEX DERIVATIVE OF SUPERIOR ORDER
}

\author{
Gabriela Cristescu, Muhammad Aslam Noor \\ AND MUHAMMAD UZAIR AWAN
}

Abstract. Some Hermite-Hadamard type inequalities via fractional integration are derived for superior order differentiable functions having one derivative with $s$-convexity of either first kind or second kind. The $n$-th order cumulative behavior of the function in the neighborhood of the frontier of the definition interval is studied in case of the $s$-convexity of second kind, by means of fractional integration. The inequalities are as best as possible from the sharpness point of view, meaning that a sharpness class of functions is identified, for each inequality, within the functions that have one derivative that is $s$-affine either of first kind or of second kind.

Mathematics subject classification (2010): 26A33, 26D15, 26 A51.

Keywords and phrases: Convex functions, Orlicz convexity, fractional integrals, Hermite-Hadamard inequality.

\section{REFERENCES}

[1] E. F. Beckenbach, Generalized convex functions, Bull. Amer. Math. Soc. 43 (1937), 363-371.

[2] W. W. BRECKNER, Stetigkeitsaussagen für eine Klasse verallgemeinerter konvexer funktionen in topologischen linearen Räumen, Publ. Inst. Math. 23 (1978) 13-20.

[3] G. CRIstescu, L. LuPŞA, Non-connected Convexities and Applications, Kluwer Academic Publishers, Dordrecht, Holland, 2002.

[4] G. Cristescu, M. A. Noor, M. U. Awan, Bounds of the second degree cumulative frontier gaps of functions with generalized convexity, Carpath. J. Math, 31 (2) (2015), 173-180.

[5] S. S. Dragomir, S. FitZPatrick, The Hadamard's inequality for s-convex functions in the first sense, Demonstratio Math., 31 (1998), (3), 633-642.

[6] S. S. Dragomir, C. E. M. Pearce, Selected Topics on Hermite-Hadamard Inequalities and Applications, Victoria University, Australia (2000).

[7] S. S. Dragomir, J. PeČArić And L. E. Persson, Some inequalities of Hadamard type, Soochow J. Math, 21 (1995), 335-341.

[8] P. M. Gruber, J. M. Willis (eds.), Handbook of Convex Geometry, A, B, North-Holland, 1993.

[9] J. HADAMARD, Étude sur les propriétés des fonctions entières et en particulier d'une fonction considerée par Riemann, J. Math. Pures Appl., 58 (1893), 171-215.

[10] Ch. Hermite, Sur deux limites d'une intégrale définie, Mathesis, 3 (1883), 82.

[11] H. HudziK, L. Maligranda, Some remarks on $s_{i}$-convex functions, Aequationes Math., 48 (1994), $100-111$.

[12] G. MAKSA, Z. PÁLES, The equality case in some recent convexity inequalities, Opuscula Math. 31 (2011), 2, 269-277.

[13] M. A. Noor, M. U. Awan, Some Integral inequalities for two kinds of convexities via fractional integrals, Trans. J. Math. Mech., 5 (2013), 2, 129-136.

[14] M. A. Noor, G. Cristescu, M. U. Awan, Generalized Fractional Hermite-Hadamard Inequalities for twice differentiable s-convex functions, Filomat, 29 (4) (2015), 807-815.

[15] W. ORLicz, A note on modular spaces I, Bull. Acad. Polon. Sci. Math. Astronom. Phys. 9 (1961), $157-162$. 
[16] B. G. PACHPATTE, On some inequalities for convex functions, RGMIA Research Report Collection, 6 (E) (2003), online: http://rgmia.vu. edu . au/v6 (E).html.

[17] M. R. Pinheiro, $H$-H inequality for S-convex Functions, International Journal of Pure and Applied Mathematics, 44 (2008), 4, 563-579.

[18] M. R. PINHEIRo, Convexity Secrets, Trafford, 2008.

[19] T. Popoviciu, Notes sur les fonctions convexes d'ordre supérieur, I, Mathematica, 12 (1936), 81-92.

[20] A. Rosenfeld, A. KaK, Digital Picture Processing, Academic Press INC., San Diego, 1976.

[21] S. Schaible, W. T. Ziemba (eds.), Generalized Convexity in Optimization and Economics, New York, Academic Press 1981.

[22] J. WAng, X. Li, M. Feckan, Y. Zhou, Hermite-Hadamard-type inequalities for RiemannLiouville fractional integrals via two kinds of convexity, Applicable Analysis, 2012, online: http://dxdoi.org/10.1080/00036811.2012.727986. 